\title{
ORIGINAL
}

\section{Ultrasound assessment of pulmonary fibroproliferative changes in severe COVID-19: a quantitative correlation study with histopathological findings}

Renata Aparecida de Almeida Monteiro' ', Amaro Nunes Duarte-Neto', Luiz Fernando Ferraz da Silva 1,2, Ellen Pierre de Oliveira ${ }^{3}$, Ellen Caroline Toledo do Nascimento ${ }^{1}$, Thais Mauad ${ }^{1}$,

Paulo Hilário do Nascimento Saldiva ${ }^{1}$ and Marisa Dolhnikoff ${ }^{*}$ (i)

(c) 2021 Springer-Verlag GmbH Germany, part of Springer Nature

\begin{abstract}
Purpose: This study was designed to evaluate the usefulness of lung ultrasound (LUS) imaging to characterize the progression and severity of lung damage in cases of COVID-19.

Methods: We employed a set of combined ultrasound parameters and histopathological images obtained simultaneously in 28 patients (15 women, 0.6-83 years) with fatal COVID-19 submitted to minimally invasive autopsies, with different times of disease evolution from initial symptoms to death (3-37 days, median 18 days). For each patient, we analysed eight post-mortem LUS parameters and the proportion of three histological patterns (normal lung, exudative diffuse alveolar damage [DAD] and fibroproliferative DAD) in eight different lung regions. The relationship between histopathological and post-mortem ultrasonographic findings was assessed using various statistical approaches.

Results: Statistically significant positive correlations were observed between fibroproliferative DAD and peripheral consolidation (coefficient $0.43, p=0.02$ ) and pulmonary consolidation (coefficient $0.51, p=0.005$ ). A model combining age, time of evolution, sex and ultrasound score predicted reasonably well $(r=0.66)$ the proportion of pulmonary parenchyma with fibroproliferative DAD.
\end{abstract}

Conclusion: The present study adds information to previous studies related to the use of LUS as a tool to assess the severity of acute pulmonary damage. We provide a histological background that supports the concept that LUS can be used to characterize the progression and severity of lung damage in severe COVID-19.

Keywords: COVID-19, Lung ultrasound, Diffuse alveolar damage, Autopsy, Minimally invasive autopsy, Pathology, Acute lung injury

*Correspondence: maridol@usp.br

1 Departamento de Patologia, Faculdade de Medicina da Universidade de São Paulo, Av. Dr. Arnaldo, 455, sala 1155, Sao Paulo, SP 01246-903, Brazil Full author information is available at the end of the article

\section{Introduction}

The current pandemic caused by SARS-CoV-2 has placed a great burden on healthcare professionals, especially those involved in the treatment of critically ill patients. In this scenario, pulmonary imaging is important to track the patients' response to treatment and disease progression [1-5].

Computed tomography (CT) is the reference imaging method for assessing pulmonary changes caused

\section{实


by coronavirus disease 2019 (COVID-19), but its use may be limited by difficulties in transporting critically ill patients with a highly contagious disease and on mechanical ventilation. The American College of Radiology recommends that computed tomography should not be used to screen or diagnose COVID-19 and that it is used sparingly in symptomatic and hospitalized patients, due to the risk of transmitting infection to staff and subsequent patients [3]. In a pandemic situation, CT platforms in referral hospitals are under high demand pressure. Due to its portability, low cost, and availability in Intensive Care Units (ICU), lung ultrasound (LUS) may represent an adequate alternative to CT for pulmonary evaluation of patients with COVID$19[6,7]$.

Based on the artifacts generated by the difference in tissue acoustic impedance consequent to reduction of pulmonary aeration or changes in tissue rheology (fibrosis, for instance), LUS exhibits specific patterns, which have been proposed as indicators of different stages of pulmonary alterations [8-10]. The first image of the pulmonary $B$ line by ultrasound was described as "an unexpected echographic aspect" by Ziskin et al. in 1982, and became the first reference to the "comet tail artefact" [11]. In 1986, Weinberg et al. demonstrated sonographic aspects of air bronchogram and since then, several studies have highlighted the use of pulmonary ultrasound [12-16]. In 1997, Lichtenstein et al. published a comparative study between ultrasound and pulmonary tomography in critically ill patients and, in 2008, the "BLUE protocol", used to characterize the acute respiratory failure in critically ill patients $[17,18]$. In 2012, Volpicelli et al. published recommendations based on international evidence on how to interpret LUS findings in critically ill patients [19].

In studies with patients with COVID-19, changes in the pleura and subpleural parenchyma have been considered as indicators, and even predictive markers, of the severity of pulmonary damage $[2,5,6]$. Following this line of reasoning, the use of LUS has been proposed to assess pulmonary involvement in hospitalized patients as well as a point-of-care screening tool $[1,4-7,20]$. The same observation has been reported in previous episodes of viral induced acute lung diseases, such as those caused by H7N9 and H1N1 influenza [3].

Histopathological studies contribute significantly to the understanding of the natural history of severe COVID-19. Initially, infection of the alveolar epithelium causes epithelial rupture, changing the permeability of the alveolar-capillary barrier, promoting diffuse alveolar damage (DAD), characterized by exudative accumulation of plasma proteins and inflammatory residues in the alveolar spaces $[8,21]$. In the most severe

\section{Take-home message}

We provide a histological background for the use of LUS as a tool to assess the progression and severity of lung damage in severe COVID-19. A model combining age, time of evolution, sex and LUS score can be used to estimate the proportion of pulmonary parenchyma with fibroproliferative changes in COVID-19-related diffuse alveolar damage.

cases, the inflammation causes progressive fibrosis of the distal air spaces, which can cause marked alveolar distortion and permanent scarring [8]. The progressive damage of gas exchange territory is the hallmark of severe COVID-19 and is an important prognostic parameter.

Our Hospital is a reference for the treatment of COVID-19 in Brazil and has allocated 300 ICU beds for severely affected patients. We established an autopsy protocol to produce information for the ICU team and to carry out mechanistic studies on the pathogenesis of COVID-19. As we do not have autopsy facilities with level 3 protection, we use an ultrasound-guided minimally invasive autopsy protocol (MIA/US) to perform post mortem examinations [21, 22]. Post-mortem ultrasonography was used to locate the organs of interest and to identify the intra-organ heterogeneity of disease, guiding tissue collection. This procedure resulted in a set of combined ultrasound parameters and histopathological images obtained during autopsy, sampled from patients with different times of disease evolution. In a previous report [23], we observed correlations between specific patterns of LUS imaging and histological changes in the pulmonary microstructure. In this study, we further explored this aspect, carrying out a systematic quantitative analysis of 28 cases.

\section{Materials and methods Population studied}

This study was approved by the HC-FMUSP Ethical Committee (protocol no. 3951.904). The procedures were performed at the Image Platform in the Autopsy Room, a research centre in the University of Sao Paulo Medical School, located next to the Autopsy Service of Sao Paulo University (https://pisa.hc.fm.usp.br/). All the autopsies were performed after informed consent had been obtained from the next of kin. We evaluated 28 patients (15 women) with ages varying from 0.6 to 83 years (median 50). The time elapsed from symptoms to death varied from 3 to 37 days (median 18 days). All patients had laboratory confirmation of SARS-CoV-2 infection by a positive RT-PCR result on the nasal/oropharyngeal swab and/or lung tissue. 


\section{Autopsy protocol}

MIA/US protocol was described previously [24] and was adapted for COVID-19 for safety reasons [21, 22]. An expert in US performed the scanning and analysis on all patients, as well as tissue collection (RAAM). Predefined reference sites of the anterior chest wall were considered for post-mortem LUS imaging and tissue sampling, considering the elevation of the diaphragmatic dome and the reduction in post-mortem lung height and volume. With the bodies in the supine position, postmortem LUS-guided tissue collection was done in eight regions, as a result of the combination of the upper and lower chest fields (lateral and medial, four sites in each lung). In each sampling site, six lung samples were collected. Such protocol resulted in 48 lung samples collected from 8 pulmonary regions for each case. We used a portable SonoSite M-Turbo R (Fujifilm, Bothell, WA, USA) with broadband and multifrequency transducers: C60x (5-2 MHz Curved) and HFL38X (13-6 MHz Linear) and $\mathrm{DICOM}^{\mathrm{TM}}$ medical images. The pulmonary evaluation was performed in the longitudinal plane with the transducer perpendicular to the cutaneous surface, adjusted in abdominal mode with a low mechanical index (0.7), which can be readjusted during the exam for better image acquisition. The examinations were conducted using predominantly the low-frequency convex transducer, which was able to produce good visualization of the pleuropulmonary interface and parenchyma. Before the autopsy, the body was packed with resistant plastic. For US orientation, small $10 \mathrm{~cm}$ openings were made to allow the direct contact between transducer and body surface. Tissue sampling was performed using Tru-Cut semi-automatic coaxial needles of 14G, $20 \mathrm{~cm}$ long. Tissue samples were fixed in buffered $10 \%$ formalin, embedded in paraffin, and stained with haematoxylin and eosin.

\section{Histological analysis}

Slides were examined by pulmonary pathologists that quantified the proportion (expressed as percentage) of alveolar tissue exhibiting three main histological patterns in each region: normal lung, exudative DAD and fibroproliferative DAD. The following histological criteria were used for defining each pattern: (a) normal lung: preserved architecture, without inflammation, oedema or exudate; (b) exudative DAD: interstitial and/or intraalveolar oedema, interstitial inflammation, variable amounts of alveolar haemorrhage and fibrin deposition, intra-alveolar hyaline membranes and type II pneumocyte hyperplasia. Foci of neutrophilic pneumonia were also included in the acute/exudative pattern; (c) fibroproliferative DAD: any degree of fibroblastic proliferation within the interstitium and/or alveolar spaces, including loose aggregates of fibroblasts admixed with scattered inflammatory cells, collagen deposition, squamous metaplasia, and possible remnants of hyaline membranes [25].

Such classification was done without knowledge of the clinical and LUS information about the cases under analysis. For each histological parameter, a mean value was obtained for the eight regions studied in each patient. This average value was used as an individual overall estimate of lung histological damage for each patient. For the analysis of the 28 patients, data are presented as mean values of each parameter. Figure 1 shows representative figures of the three histological patterns considered in the present study.

\section{Ultrasound analysis}

We defined for each of the eight sites of study a binary indicator $(0=$ absence; $1=$ presence $)$ of LUS parameters previously described in several studies dedicated to assess the severity of pulmonary COVID-19 [2, 5, 26], as follows: normal pleural line, irregular pleural line, fragmented pleural line, A lines, spaced B lines, confluent $\mathrm{B}$ lines (sometimes giving the appearance of a white lung), peripheral consolidation, and pulmonary consolidation. For each patient, values of LUS parameters were calculated as the sum of the scores obtained at the eight examined sites (therefore, the value of each LUS parameter varied from 0 to 8 for each patient). Figure 2 shows a panel of representative aspects of LUS parameters evaluated in this study. Pulmonary impairment in COVID-19 presents itself as a mixture of patterns observed in LUS, which vary according to disease's severity. Thus, depending on the analysed region, LUS may disclose several or even all of the patterns described above in the same patient.

\section{Statistical analysis}

The relationship between histopathological and ultrasonographic findings was assessed using various statistical approaches. We compared the ultrasound parameters in groups with different intensities of pulmonary histological damage using non-parametric statistics. Multivariate regression analysis was employed to verify the association between pulmonary damage and US scores, adjusted for age, sex, and time of clinical evolution. Statistical analyses were done with the aid of the SPSS V25 software.

\section{Results}

Table 1 shows the epidemiological and clinical data of the 28 studied patients. The patients comprised 15 women and 13 men, with a median age of 50 years (range, 0.683 years). The median timespan between the occurrence 


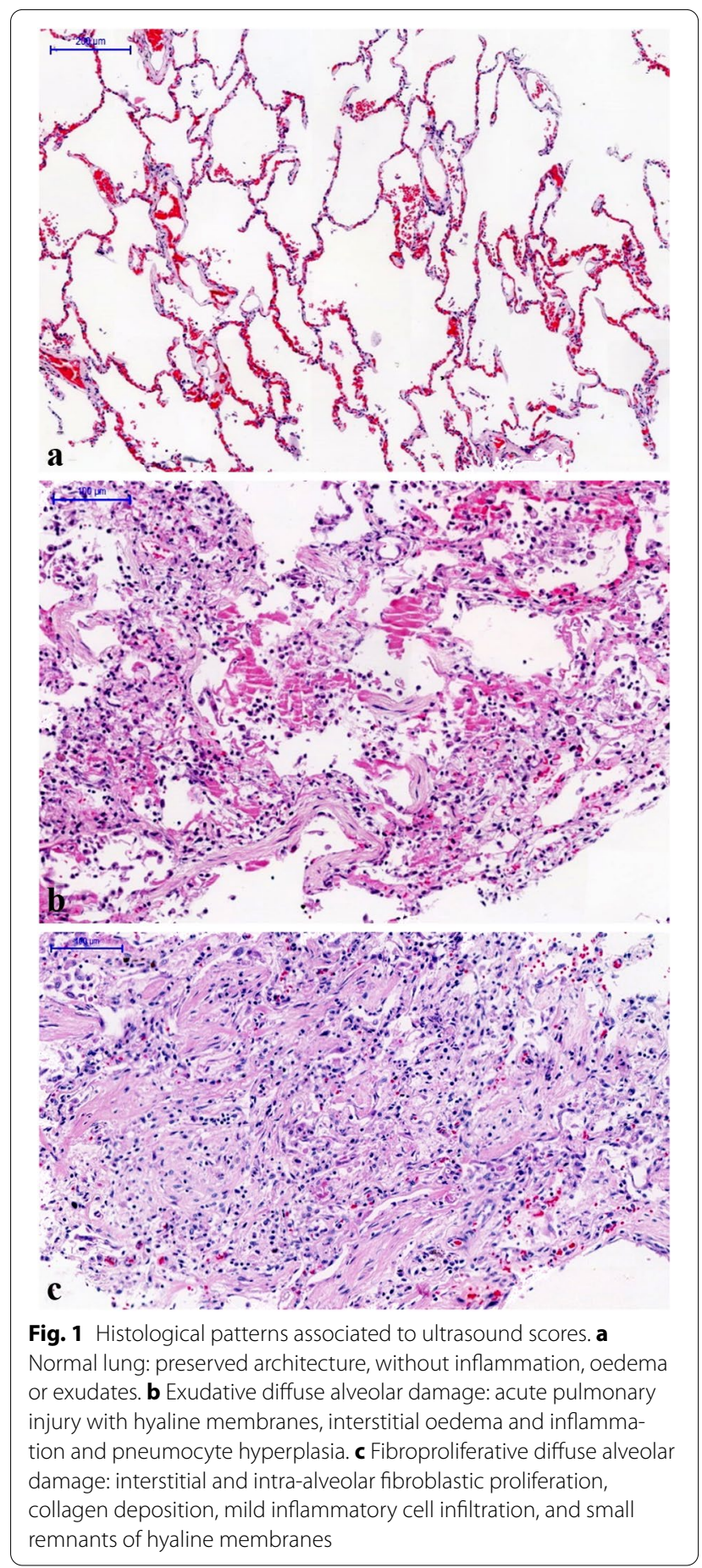

of symptoms and death was 18 days (range, 3-37 days). The most frequent associated clinical conditions were diabetes mellitus, systemic arterial hypertension, and chronic ischaemic cardiopathy. A 7-month-old patient had Edwards syndrome. All patients presented with severe respiratory distress and showed diffuse alveolar damage on lung histopathological analysis, with varying degrees of organization. Table 2 presents the descriptive statistics of the histopathological and LUS parameters. Depth of peripheral consolidations varied from 0.5 to $2.1 \mathrm{~cm}$ (median $=1.2 \mathrm{~cm}$ ). Statistically significant positive correlations (Spearman correlation) were observed between fibroproliferative DAD and peripheral consolidation (coefficient 0.43, $p=0.023$ ) and pulmonary consolidation (coefficient $0.51, p=0.005$ ). Pulmonary consolidation exhibited a negative and significant correlation with mean proportion of normal alveolar parenchyma (coefficient $-0.4, p=0.037$ ). No significant associations were detected for any LUS parameter and exudative DAD. Considering that peripheral consolidation and pulmonary consolidation varied with the extent of the fibroproliferative alveolar process, we produced a LUS severity score (LUSCORE) for each patient, computed as the sum of these two parameters, including all analysed regions (therefore, values of LUSCORE ranged from 0 to 16 in each patient).

Figure 3 shows the variation of the proportions of histological parameters among the 28 patients, disaggregated by tertiles of LUSCORE. The proportion of fibroproliferative DAD exhibited significant differences across the three LUSCORE tertiles $(p=0.02$, Kruskal-Wallis statistics). Post hoc comparison (ANOVA on Ranks) indicated that the proportion of pulmonary parenchyma with fibroproliferative DAD was significantly higher in the third tertile of LUSCORE in comparison with those in the first tertile $(p=0.013)$, indicating that patients with higher LUS severity scores present higher proportion of fibroproliferative DAD.

Multiple linear regression analysis considered the proportion of fibroproliferative DAD as the dependent variable and time of disease evolution, LUSCORE, age and a binary indicator of sex $(0=$ males, $1=$ females $)$ as predictive parameters. Age (coefficient 0.68, $p=0.024$ ) and LUSCORE (coefficient 6.75, $p=0.009$ ) were positively and significantly associated with the extent of fibroproliferative DAD (adjusted $r$ value $=0.66$ ). Time between symptoms' onset and death (coefficient $=0.655, p=0.28$ ) and sex (female, coefficient $=-7.83, p=0.48$ ) did not show significant associations in this model. Our results indicate that the extent of fibroproliferative DAD may be estimated by the following equation:

$$
\begin{aligned}
& \text { Fibroproliferative DAD }(\%) \\
& \begin{aligned}
= & -18.06+\text { age }(\text { years }) * 0.682 \\
& +\operatorname{sex}(F=1, M=0) *-7.830 \\
& + \text { time }(\text { days }) * 0.655+\text { LUSCORE } * 6.754 .
\end{aligned}
\end{aligned}
$$




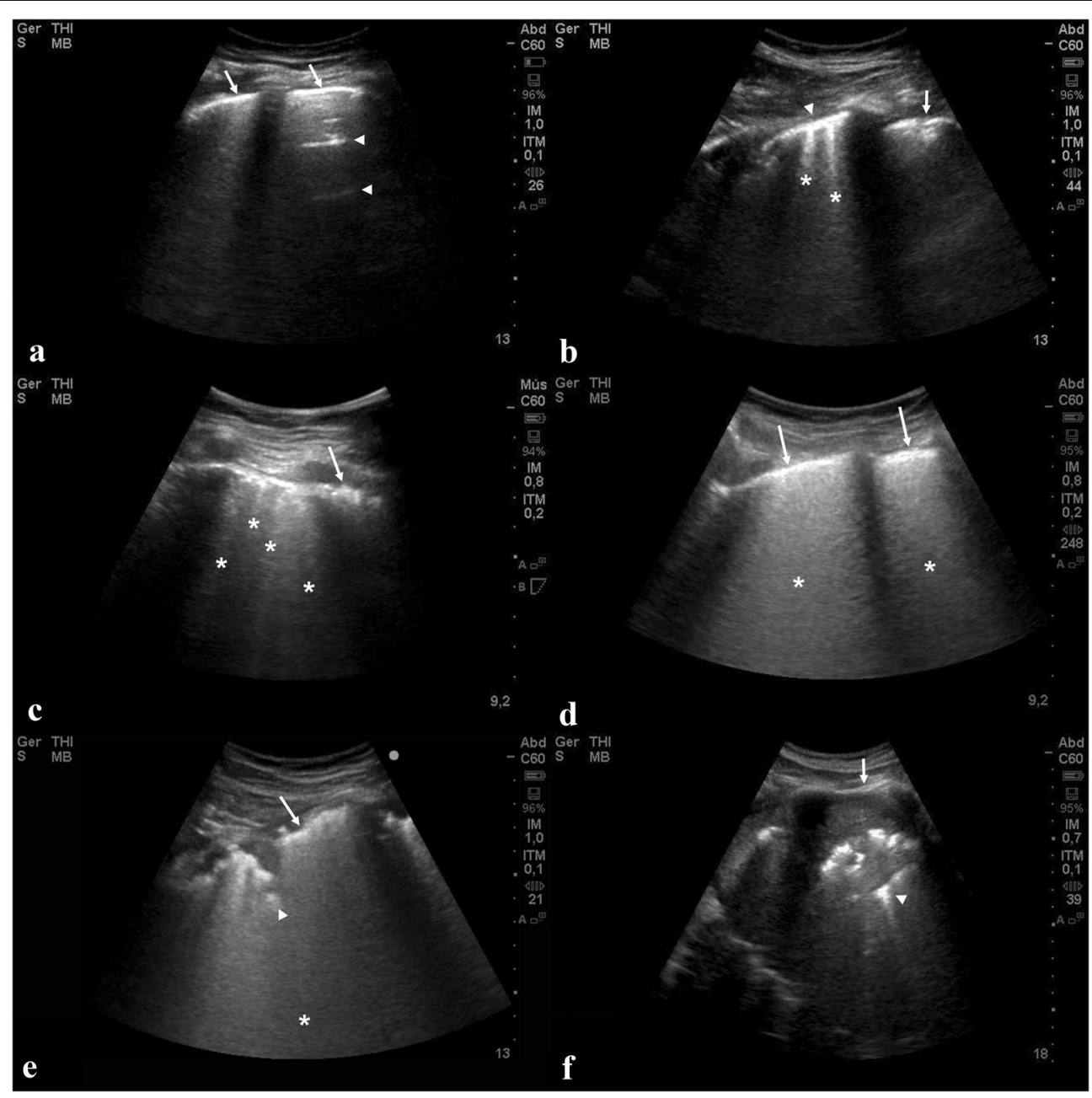

Fig. 2 The several patterns of LUS in COVID-19 patients: a normal pleural line (arrows) and A lines (arrowheads). b normal pleural line (arrowhead), spaced B lines (asterisks), and fragmented pleural line (arrow). c irregular pleural line (arrow) and confluent B lines (asterisks). d normal pleural line (arrows) and white lung (asterisks). e irregular pleural line (arrow), peripheral consolidation (arrowhead), and white lung (asterisk). f normal pleural line (arrow) and pulmonary consolidation with air bronchogram (arrowhead)

\section{Discussion}

Several studies on the use of LUS in COVID-19, using CT imaging as reference standard, have indicated that LUS may be useful to characterise the severity of pulmonary injury $[2,6,27]$. We further explored the usability of LUS in COVID-19, considering histopathological aspects as reference to quantify pulmonary damage. We employed a set of combined LUS parameters and histopathological images obtained simultaneously at autopsy of 28 patients.

In fact, such combination-LUS + histopathology-is rare in the clinical environment, and is only possible in autopsy or experimental settings [21]. Recently, neonatal lung US was validated as a sensitive indicator of acute respiratory distress syndrome in a neonatal lung injury swine model, with a moderate degree of correlation between in vivo US observation and the degree of histological injury [28]. In a rabbit ALI model, the frequency of post-mortem B-line artifact in ex vivo US correlated closely with the severity of pulmonary histological abnormalities [29]. We are not aware of any study that has compared pre-mortem with post-mortem LUS findings, and pre-mortem LUS was not performed on our patients. Therefore, for the post-mortem analysis, we used LUS parameters previously established in vivo [2, 3, 5, 7, 26]. To our knowledge, this is the first study to correlate ultrasound imaging with histological injury in COVID-19 patients; our results provide additional information to the previous in vivo imaging studies and reinforce the concept that LUS is 
Table 1 Epidemiological and clinical data of 28 patients with fatal COVID-19

\begin{tabular}{ll}
\hline Characteristic & Patients (n) \\
\hline Female/male & $15 / 13$ \\
Age in years, median (range) & $50(0.6-83)$ \\
Comorbidities, $n$ (\%) & $10(35.71 \%)$ \\
\hline Diabetes & $9(32.14 \%)$ \\
\hline Systemic arterial hypertension & $9(32.14 \%)$ \\
\hline Ischemic cardiopathy & $18(64.28 \%)$ \\
\hline Symptoms, $n$ (\%) & $22(78.57 \%)$ \\
\hline Fever & $19(67.85 \%)$ \\
\hline Dyspnoea & $6(21.43 \%)$ \\
\hline Cough & $6(21.43 \%)$ \\
\hline Rhinorrhea & $11(39.28 \%)$ \\
\hline Diarrhoea & $18(3-37)$ \\
\hline Myalgia & $11.5(0-34)$ \\
\hline Time from symptom onset to death in days, median (range) & $8.5(0-25)$ \\
\hline Period of hospitalisation in days, median (range) & $10.5(0-31)$ \\
\hline Intensive care unit stay in days, median (range) & \\
\hline Period of mechanical ventilation in days ( $n=26$ cases), median (range) & \\
\hline
\end{tabular}

Table 2 Descriptive statistics of the histopathological and LUS parameters for the 28 patients

\begin{tabular}{|c|c|c|c|c|}
\hline Lung parameter & Minimum & Maximum & Mean & SD \\
\hline \multicolumn{5}{|l|}{ Histopathological parameters (\%) } \\
\hline Normal lung tissue & 0 & 85 & 24.64 & 27.06 \\
\hline Exudative diffuse alveolar damage & 0 & 100 & 36.05 & 29.89 \\
\hline Fibroproliferative diffuse alveolar damage & 0 & 100 & 37.81 & 35.37 \\
\hline \multicolumn{5}{|l|}{ Lung ultrasound parameters } \\
\hline Normal pleural line & 0 & 7 & 0.29 & 1.33 \\
\hline Irregular pleural line & 0 & 8 & 2.54 & 2.44 \\
\hline Fragmented pleural line & 0 & 4 & 0.68 & 1.25 \\
\hline A lines & 0 & 3 & 1.04 & 1.14 \\
\hline B lines & 0 & 7 & 1.57 & 1.69 \\
\hline Confluent B lines & 0 & 8 & 5.32 & 2.87 \\
\hline Peripheral consolidation & 0 & 6 & 1.18 & 1.76 \\
\hline Pulmonary consolidation & 0 & 6 & 0.82 & 1.44 \\
\hline
\end{tabular}

Minimum, maximum, mean and standard deviation (SD) values for the 28 patients. Histological parameters: For each patient, the mean proportion (\%) of each parameter was obtained for the eight studied regions. LUS parameters: For each patient, we used the sum of the scores in each of the eight regions

accurate enough to track the extent of fibroproliferative alveolar damage in severe COVID-19.

Our analyses detected significant associations between some LUS parameters and progression of pulmonary fibrosis, a frequent complication of diffuse alveolar damage [8]. In fact, ultrasound techniques have already been used to determine the extent of fibrosis in other organs, based on the differences on the stress/strain properties of fibrotic tissue in respect to normal parenchyma [3032]. Our results indicate that LUS can track the extent of pulmonary fibrosis as well, a finding that may improve the adequate management of critical patients, since pulmonary fibroproliferation is a significant marker of the severity of COVID-19.

Previous reports indicate that the extent of pulmonary fibrosis is associated with poor prognosis and also may cause permanent damage to patients that survive the severe forms of the disease, a situation that may promote a significant loss of quality of life after discharge from the ICU [33-35]. Our results suggest that non-invasive 

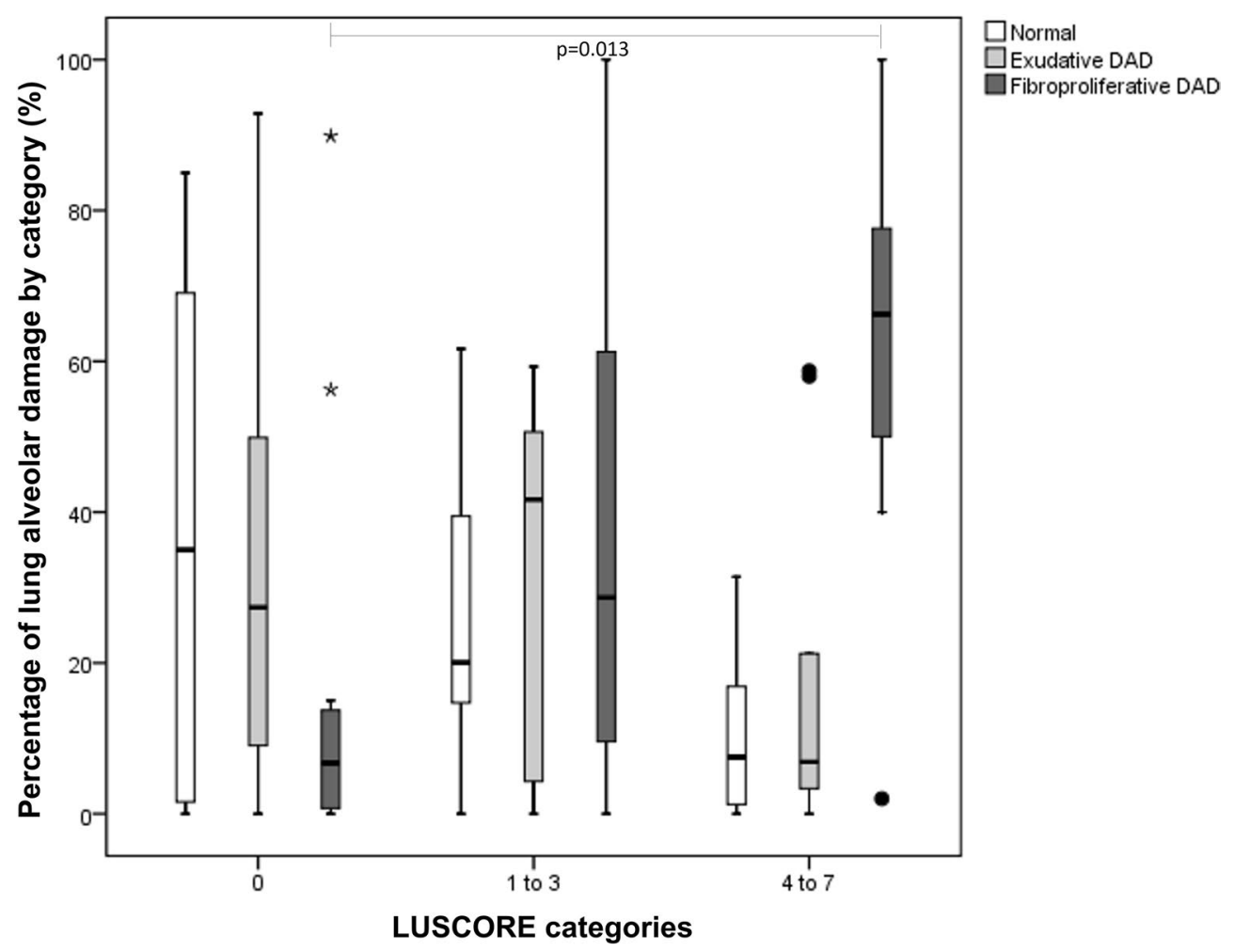

Fig. 3 Box plots represent the variation of the proportion (\%) of normal alveolar parenchyma, exudative diffuse alveolar damage (DAD) and fibroproliferative DAD among the 28 patients, disaggregated by LUSCORE tertiles

ultrasound parameters, in combination with simple clinical information, may be used to estimate the extent of pulmonary fibrosis in COVID-19, indicating that LUS may be used for sequential evaluations of the severity of pulmonary damage, with minimal risk for patients and staff.

LUS parameters significantly associated with fibroproliferation were those related to parenchymal consolidation (peripheral consolidation and pulmonary consolidation). These findings are in line with the studies that used LUS scores for the evaluation of patients with COVID-19 pneumonia. As part of the categories proposed by Volpicelli et al. for assessing the probability of the disease, the finding of multiple small peripheral consolidations is associated with a high probability of COVID-19. Nouvenne et al. identified four possible changes in LUS on hospital admission of patients with COVID-19 pneumonia: focal areas of nonconfluent B lines, diffuse confluent $B$ lines, small subpleural microconsolidations with pleural line irregularities, and large parenchymal consolidations with air bronchograms [36]. Other studies that proposed standardization with respect to the use of LUS in the treatment of patients with COVID-19 have also included the findings of peripheral and parenchymal consolidation in their scores of
COVID-19 severity [2, 20,37]. Considering the different scores used in LUS assessment, we can infer that some parameters stand out as indicators or predictors of diagnosis probability and disease severity: in screening, small peripheral consolidations are associated with a high probability of the disease, and in hospitalized patients, the peripheral and parenchymal consolidation have been associated with the severity of lung damage $[2,6,20,36$, 37].

We did not find significant associations between any LUS parameter and exudative DAD. We believe that our case series is not ideal for analysing the most acute phase of the disease. As we were only dealing with severe cases with fatal outcome, most patients had mixed patterns of lung injury, with varying amounts of fibroproliferative changes; exclusively acute changes were not frequently seen. In this context, rather than identifying the acute phase of the disease, the LUS score we propose is more suitable for tracking the intensity of fibroproliferative changes in severe COVID-19.

Although fibroproliferative changes tended to be more prevalent in late stages of the disease, we did not observe a significant association between the duration of the disease and the proportion of fibroproliferative DAD. Histopathological analysis showed that the amount 
of fibroproliferative changes varied widely among individuals, even after prolonged hospitalization. The lack of association between the duration of the disease and the intensity of fibroproliferative changes, and the fact that some patients did not have severe fibrosis in late stages, suggests that some individuals with COVID-19-related pneumonia may not progress to a fibroproliferative stage.

Our study has some important limitations; first, our series is relatively small, and larger series are needed to confirm the present findings and associations. Second, pre-mortem LUS was not performed on our patients and the post-mortem LUS does not necessarily reflect the exact same aspects of living patients. Differences between in vivo and post-mortem LUS images may exist since some retraction of pulmonary parenchyma occurs after death. Thus, not only larger autopsy studies, but also clinical validation investigations are needed to strengthen the plausibility of our results.

In conclusion, the present study adds information to previous studies related to the use of LUS as a tool to assess the severity and progression of acute pulmonary damage. Our results provide a histological background that supports the concept that LUS can be used to track the extent of fibroproliferative DAD in severe COVID-19.

\begin{abstract}
Author details
${ }^{1}$ Departamento de Patologia, Faculdade de Medicina da Universidade de São Paulo, Av. Dr. Arnaldo, 455, sala 1155, Sao Paulo, SP 01246-903, Brazil. ${ }^{2}$ Serviço de Verificação de Óbitos da Capital, Universidade de São Paulo, Sao Paulo, Brazil. ${ }^{3}$ Departamento de Cardiopneumologia, Instituto do Coração, Faculdade de Medicina da Universidade de São Paulo, Sao Paulo, Brazil.
\end{abstract}

\begin{abstract}
Acknowledgements
The authors wish to thank Reginaldo Silva do Nascimento, Jair Theodoro Filho, Thábata Larissa Luciano Ferreira Leite and Catia Sales de Moura for their technical support. We thank all technicians working in the histopathology laboratory of the Department of Pathology-FMUSP. We are grateful to those involved in the care of patients with COVID-19; all hospital workers who participated in the HC-FMUSP Coronavirus Crisis Committee during the epidemic season, and all legal representatives who agreed to the post-mortem examination of patients with COVID-19.
\end{abstract}

\section{Author contributions}

The study was designed by RAAM and PHNS. RAAM performed LUS scanning and analysis, and tissue collection; ANDN, LFFS, ECTN, TM, PHNS and MD performed the histological analysis; EPO collected the clinical data. Statistical analyses were performed by PHNS. The first draft of the manuscript was written by PHNS, RAAM, MD and ANDN, and all authors commented on previous versions of the manuscript. All authors read and approved the final manuscript.

\footnotetext{
Funding

Fundação de Amparo à Pesquisa do Estado de São Paulo (Grant Number: 2013/17159-2, https://doi.org/10.13039/501100001807), Bill and Melinda Gates Foundation (Grant Number: INV-002396, https://doi.org/10.13039 /100000865) and Conselho Nacional de Desenvolvimento Científico e Tecnológico 304987/2017-4
}

\section{Compliance with ethical standards}

Conflicts of interest

The authors declare that they have no conflicts of interest.

\section{Ethical approval}

This study was approved by the HC-FMUSP Ethical Committee (Protocol No. 3951.904)

\section{Publisher's Note}

Springer Nature remains neutral with regard to jurisdictional claims in published maps and institutional affiliations.

Received: 4 September 2020 Accepted: 28 November 2020

Published online: 3 January 2021

\section{Reference}

1. Smith MJ, Hayward SA, Innes SM, Miller ASC (2020) Point-of-care lung ultrasound in patients with COVID-19 - a narrative review. Anaesthesia. https://doi.org/10.1111/anae.15082

2. Pata D, Valentini P, De Rose C, De Santis R, Morello R, Buonsenso D (2020) Chest computed tomography and lung ultrasound findings in COVID-19 pneumonia: a pocket review for non-radiologists. Front Med. https://doi. org/10.3389/fmed.2020.00375

3. Convissar DL, Gibson LE, Berra L, Bittner EA, Chang MG (2020) Application of lung ultrasound during the COVID-19 pandemic: a narrative review. Anesth Analg. https://doi.org/10.1213/ANE.0000000000004929

4. Guarracino F, Vetrugno L, Forfori F et al (2020) Lung, heart, vascular, and diaphragm ultrasound examination of COVID-19 patients: a comprehensive approach. J Cardiothorac Vasc Anesth. https://doi.org/10.1053/j. jvca.2020.06.013

5. Moore S, Gardiner E (2020) Point of care and intensive care lung ultrasound: a reference guide for practitioners during COVID-19. Radiography. https://doi.org/10.1016/j.radi.2020.04.005

6. Volpicelli G, Lamorte A, Villén T (2020) What's new in lung ultrasound during the COVID-19 pandemic. Intensive Care Med. https://doi.org/10.1007/ s00134-020-06048-9

7. Kulkarni S, Down B, Jha S (2020) Point-of-care lung ultrasound in intensive care during the COVID-19 pandemic. Clin Radiol. https://doi. org/10.1016/j.crad.2020.05.001

8. Istvan-Adorjan S, Ágoston G, Varga A, Cotoi OS, Frigy A (2020) Pathophysiological background and clinical practice of lung ultrasound in COVID-19 patients: a short review. Anatol J Cardiol. https://doi.org/10.14744/Anato JCardiol.2020.33645

9. Hubmayr RD, Kallet RH (2018) Understanding pulmonary stress-strain relationships in severe ARDS and its implications for designing a safer approach to setting the ventilator. Respir Care. https://doi.org/10.4187/ respcare.05900

10. Goffi A, Kruisselbrink R, Volpicelli G (2018) The sound of air: point-of-care lung ultrasound in perioperative medicine. Le bruit de l'air : échographie pulmonaire au point d'intervention en médecine périopératoire. Can J Anaesth. https://doi.org/10.1007/s12630-018-1062-x

11. Ziskin MC, Thickman DI, Goldenberg NJ, Lapayowker MS, Becker JM (1982) The comet tail artifact. J Ultrasound Med. https://doi.org/10.7863/ jum.1982.1.1.1

12. Weinberg B, Diakoumakis EE, Kass EG, Seife B, Zvi ZB (1986) The air bronchogram: sonographic demonstration. AJR Am J Roentgenol. https ://doi.org/10.2214/ajr.147.3.593

13. Lichtenstein D, Mezière G, Biderman P, Gepner A (1999) The comet-tail artifact: an ultrasound sign ruling out pneumothorax. Intensive Care Med. https://doi.org/10.1007/s001340050862

14. Lichtenstein DA, Lascols N, Mezière G, Gepner A (2004) Ultrasound diagnosis of alveolar consolidation in the critically ill. Intensive Care Med. https://doi.org/10.1007/s00134-003-2075-6

15. Lo Giudice V, Bruni A, Corcioni E, Corcioni B (2008) Ultrasound in the evaluation of interstitial pneumonia. J Ultrasound. https://doi.org/10.1016/j. jus.2007.10.002

16. Volpicelli G, Caramello V, Cardinale L, Mussa A, Bar F, Frascisco MF (2008) Detection of sonographic B-lines in patients with normal lung or radiographic alveolar consolidation. Med Sci Monit 14(3):CR122-CR128 
17. Lichtenstein D, Mézière G, Biderman P, Gepner A, Barré O (1997) The comet-tail artefact. An ultrasound sign of alveolar-interstitial syndrome. Am J Respir Crit Care Med. https://doi.org/10.1164/ajrccm.156.5.96-07096

18. Lichtenstein DA, Mezière GA (2008) Relevance of lung ultrasound in the diagnosis of acute respiratory failure: the BLUE protocol. Chest. https:// doi.org/10.1378/chest.07-2800

19. Volpicelli G, Elbarbary M, Blaivas M, Lichtenstein DA, Mathis G, Kirkpatrick AW et al (2012) International Liaison Committee on Lung Ultrasound (ILC-LUS) for International Consensus Conference on Lung Ultrasound (ICC-LUS). International evidence-based recommendations for point-ofcare lung ultrasound. Intensive Care Med. https://doi.org/10.1007/s0013 4-012-2513-4

20. Smargiassi A, Soldati G, Borghetti A et al (2020) Lung ultrasonography for early management of patients with respiratory symptoms during COVID19 pandemic. J Ultrasound. https://doi.org/10.1007/s40477-020-00501-7

21. Nunes Duarte-Neto $A$, de Almeida Monteiro RA, da Silva LFF et al (2020) Pulmonary and systemic involvement of COVID-19 assessed by ultrasound-guided minimally invasive autopsy. Histopathology. https:// doi.org/10.1111/his.14160

22. Monteiro RAA, Duarte-Neto AN, Silva LFFD et al (2020) Ultrasoundguided minimally invasive autopsies: a protocol for the study of pulmonary and systemic involvement of COVID-19. Clinics. https://doi. org/10.6061/clinics/2020/e1972

23. Almeida Monteiro RA, de Oliveira EP, Nascimento Saldiva PH, Dolhnikoff M, Duarte-Neto AN, BIAS - Brazilian Image Autopsy Study Group (2020) Histological-ultrasonographical correlation of pulmonary involvement in severe COVID-19. Intensive Care Med. https://doi.org/10.1007/s0013 4-020-06125-z

24. Duarte-Neto AN, Monteiro RAA, Johnsson J et al (2019) Ultrasoundguided minimally invasive autopsy as a tool for rapid post-mortem diagnosis in the 2018 Sao Paulo yellow fever epidemic: correlation with conventional autopsy. PLoS Negl Trop Dis. https://doi.org/10.1371/journ al.pntd.0007625

25. Cheung O-Y, Graziano P, Smith ML (2018) Acute lung injury. In: Leslie KO, Wick MR (eds) Practical pulmonary pathology: a diagnostic approach, 3rd edn. Elsevier, Amsterdam, pp 125-146

26. Volpicelli G, Gargani L (2020) Sonographic signs and patterns of COVID-19 pneumonia. Ultrasound J. https://doi.org/10.1186/s13089-020-00171-w
27. Di Serafino M, Notaro M, Rea G et al (2020) The lung ultrasound: facts or artifacts? In the era of COVID-19 outbreak. Radiol Med. https://doi. org/10.1007/s11547-020-01236-5

28. Elsayed YN, Hinton M, Graham R, Dakshinamurti S (2020) Lung ultrasound predicts histological lung injury in a neonatal model of acute respiratory distress syndrome. Pediatr Pulmonol. https://doi.org/10.1002/ppul.24993

29. Zhu Z, Lian X, Zeng Y et al (2019) Point-of-care ultrasound-a new option for early quantitative assessment of pulmonary edema. Ultrasound Med Biol. https://doi.org/10.1016/j.ultrasmedbio.2019.08.008

30. Sigrist RMS, Liau J, Kaffas AE, Chammas MC, Willmann JK (2017) Ultrasound elastography: review of techniques and clinical applications. Theranostics. https://doi.org/10.7150/thno.18650

31. Rjosk-Dendorfer D, Reichelt A, Clevert D (2014) Elastographie als Zusatzmodalität der Mammasonographie. Radiologe. https://doi.org/10.1007/ s00117-013-2575-5

32. Balleyguier C, Canale S, Ben Hassen W et al (2013) Breast elasticity: principles, technique, results: an update and overview of commercially available software. Eur J Radiol. https://doi.org/10.1016/j.ejrad.2012.03.001

33. Leo F, Wormanns D, Grohé C (2020) COVID-19 aus Sicht der Pneumologie - Langzeitfolgen und Implikationen für die pneumologische Nachsorge [COVID-19: a pneumological point of view - long-term sequelae of COVID-19 - implications for follow-up in respiratory medicine]. Dtsch Med Wochenschr. https://doi.org/10.1055/a-1164-4040

34. George PM, Wells AU, Jenkins RG (2020) Pulmonary fibrosis and COVID19: the potential role for antifibrotic therapy. Lancet Respir Med. https:// doi.org/10.1016/S2213-2600(20)30225-3

35. Ojo AS, Balogun SA, Williams OT, Ojo OS (2020) Pulmonary fibrosis in COVID-19 survivors: predictive factors and risk reduction strategies. Pulm Med. https://doi.org/10.1155/2020/6175964

36. Nouvenne A, Zani MD, Milanese G et al (2020) Lung ultrasound in COVID19 pneumonia: correlations with chest CT on hospital admission. Respiration. https://doi.org/10.1159/000509223

37. Soldati G, Smargiassi A, Inchingolo R et al (2020) Proposal for international standardization of the use of lung ultrasound for patients with COVID-19: a simple, quantitative, reproducible method. J Ultrasound Med. https:// doi.org/10.1002/jum.15285 